\title{
CULTURE ARTISTIQUE ET ART CONTEMPORAIN
}

Les grandes tendances de l'art contemporain invitent dans leurs convergences étonnantes a renouveler notre image de la culture artistique. Quasi-mysticisme des artistes <<visionnaires $>>$, ou jeux de l'esprit des démarches dada, se rejoignent au delà des contrastes, pour rompre avec l'héritage artistique, le savoir infaillible, les voies usuelles de communication. La <<perception aigüe d'une incompréhensibilité éternelle >> ou le gout de la subversion peuvent-ils s'enseigner? Qu'en est-il de notre désir de comprendre et de faire comprendre de telles démarches?

\begin{abstract}
"Est-ce que l'artiste doit se concentrer sur une oeuvre qui serait une sorte d'accumulateur d'énergie spirituelle où est-ce qu'il doit être une sorte de missionnaire qui, par son charisme, amènerait les gens à ouvrir en eux les sources d'ou viennent les ceuvres d'art. Si l'oeuvre est menacté d'un côté, de dégenérer en pur objet esthétique, en décor de la vie, quelque chose comme un immeuble ou un vêtement; si elle court de l'autre côte le risque de se voir réduite à un geste excentrique, ce n'est pas sa faute. Cela résulte plutot de sa situation dans le monde moderne: notre civilisation n'a pas de place pour l'art dans ses structures mentales et elle ne peut donc en avoir non plus dans ses structures materielles. On ne sait donc ou mettre l'oeuvre d'art'".
\end{abstract}

'Pourtant, il doit bien avoir une issue. Notre civilisation n'a pu devenir inhumaine au point de ne plus avoir besoin d'art. Les artistes ne sont pas au monde pour rien. Il faut bien qu'ils finissent par rencontrer quelque part leurs contemporains."

Art et culture, "Culture artistique", et "culture," celle-ci entendue au sens le plus large d'une constellation de coutumes, croyances, langues, idées, gonts esthétiques, connaissances scientifiques et techniques et organisation matérielle d'un environnement transmissible.

Même si l'acception au singulier de ces concepts de Culture ou d'Art, résulte d'une sommation hative de caractéristiques amalgamées les unes aux autres, nous ne pouvons éviter de poser le probleme de leurs rapports en termes de parenté ou de rupture.

\footnotetext{
'Jindrich Chalupecky, Postface aux "histoires." Prague: 1984.
} 
1 - Parenté, filiation d'abord: on a longtemps inscrit l'art et les règles esthétiques dans un contexte culturel plus large dont ils étaient le "contenu sédimenté." Erwin Panovsky montre comment la géométrie projective était un produit des ateliers d'artistes et comment la production artistique résumait aussi les connaissances d'une époque. Francastel disait de la peinture figurative qu'elle fixait le lieu imaginaire où se hierarchisent des experiences individuelles et collectives et ou les hommes d'une sociétée donnée anticipent leurs possibilités de transformation du monde en stabilisant certains aspects.

2 - Aucune oeuvre cependant n'existerait sans une réaclion vive et curieuse, sans le choc, dit Segalen, d'une inclividualite forte contre une objectivité dont elle peréoit et déguste la distance. L'artiste est un exote et les sensations d'exotisme et l'individualisme sont complémentaires. Les oeuvres ne sont pas rares dans l'art moderne qui se sont développées sous le signe de la transgression, de la volonté de brouiller les frontières et d'abolir les contradictions.

La premiere tâche d'un artiste est de détruire; nier ce qui le précède, douter de son propre sens ou mettre en cause cette culture dans laquelle on prétend le situer. Le reste viendra par surcroît ou ne viendra pas.

Le peintre vraiment témoin de son temps n'est pas Buffet ou Brayer, David ou Meissonnier mais celui qui nous met à la question, qui se constitue comme question.

"Esprits d'angle," ecrivait Michaux, esprits tendus vers leur révolte.

Et parce que nous avons appris de $\mathrm{M}$. Foucault l'importance des métaphores géographiques et géologiques dans la pensée contemporaine, nous dirons qu'une oeuvre est actuelle quand elle montre comment le sol de la culture s'effrite et s'effondre, quand nous nous sentons contestés.

On pense, bien sûr, aux espaces vides, déchirés, à ces corps morcelés qui renvoient à notre société creuse, écartelée, en désignant une béance au centre et au fondement de notre être. Mais on pense aussi par exemple à l'effervescence du début du siècle, à la prolifération des ismes," a ces artistes du Blaue Reiter, Franz Marc et V. Kandinsky qui ont renouvelé complètement notre façon de penser l'oeuvre d'art et la culture artistique. 
Entrer dans la sphère de l'art pour Kandinsky, c'est passer d'un tableau votif à un Delaunay, d'un Cézanne à un dessin populaire russe, d'un masque africain à un Picasso et en eprouver des vibrations. L'image d'une correspondance entre les arts était au debut du siècle une conviction commune aux peintres et aux musiciens, a Schoenberg et a Kandinsky. Vieille idee du romantisme allemand, qu'on trouvait déja chez Platon, la "synesthesie" des arts pourrait faire l'objet d'une réflexion pédagogique originale. Quatre-vingts ans se sont écoulés depuis le Blaue Reiter et nous ne partageons plus l'optimisme selon lequel le monde se dirige vers une nouvelle époque du spirituel. Les idées de Kandinsky sont bien l'expression d'une époque.

Tout au contraire, un certain consensus se dégage de la certitude que notre culture est le royaume du tiède (Segalen) ou du bouillon de culture (Lussato), de l'invasion de la barbarie, de la sous-culture de masse. Et si la tension exotique decroit avec la chute des barrières et les grands raccourcis d'espaces, comment pouvons-nous retrouver le sens de la reaction vive et curieuse devant des oeuvres appartenant à des cultures ou des époques différentes, cette aptitude à sentir toute la saveur du divers, qu'évoquait déjà Segalen en 1904?

Les touristes et les géographes sont nombreux aujourd'hui, qui se reconnaissent à ce qu'à travers les pires vitesses et les pires éloignements, ils retrouvent "leurs bas de laine, leurs économies et leurs sieste ${ }^{2}$.

A cette question qui, au fond se ramène à la question de la place de la disposition esthétique et de l'amour de l'art dans le contexte culturel aujourd'hui, nous pouvons tenter de répondre en présentant la convergence étonnante de démarches artistiques qui, à notre sens, ont posé un certain nombre de questions philosophiques, existentielles ouvrant des perspectives pédagogiques neuves. On ne peut certes toutes les aborder. Mais le probleme de tout l'art moderne apparaît nettement a travers deux orientations distinctes et contradictoires qui se rejoignent dans le désir "de rompre le,charme esthétique" (J. Chalupecky), de faire un art, qui d'une maniere ou d'une autre se remplisse de vie.

D'un côté, des démarches inaugurees par Dada et la question radicale de la pertinence de la hierarchie des valeurs qui hante l'art

${ }^{2} V$. Segalen. Essai sur l'exotisme. 
contemporain et nous incite à réfléchir sur la nécessité de préserver ou non des lignes de démarcation.

De l'autre, la volonté persistante de conserver l'intégrité matérielle de l'oeuvre fermée sur elle-même et d'en travailler la structure afin qu'elle puisse intégrer la plus large experience possible de l'origine, de l'absolu, tout en interrogeant l'origine du geste, de la forme. Peintres, Poètes, Musiciens mystiques ou philosophes sans être tout à fait ni l'un ni l'autre.

Deux manières de rencontrer les fondements aux confins de l'art: l'exotisme de l'inhumain, de l'universel des artistes visionnaires ou l'exotisme du regard froid, du deplacement du regard. Sommes-nous capables de suivre ces artistes pour ouvrir en nous les sources d'où viennent les oeuvres d'art?

Si nous commençons par souligner les convergences étonnantes dans les démarches artistiques des peintres, musiciens, poètes qui font retour à l'essentiel (la lumiere, l'espace, l'Un, silence) c'est que leur exotisme à été rapproché par bien des auteurs (cf. Kadinsky ou Segalen) de celui de l'enfant avant l'irruption de l'histoire et de la geographie. Tobey, Rothko, Newman, Reinhart, Klein, Michaux, Pollock. Que reste-til aujourd'hui de leur embrasement!

Quelques clichés. L'infinie diversité de la culture rend sans doute extrêmement improbable la construction de grandes pensées, de telles institutions directrices.

\section{Les "vigionnaires"}

L'artiste que l'on a considéré comme un maître spirituel n'est sans doute pas forcément un "abstrait", et dans l'extraordinaire effervescence des "ismes" au début du siècle, tous les artistes dans leur refus de la peinture figurative se concentrent sur l'"essentiel" en tournant le dos à l'histoire.

Mais ce sont les peintres abstraits, ceux qui aboutirent à une vision consciente et non à une impulsion passagere de l'abstraction, qui écrivent pour convaincre que le tableau acquiert le pouvoir de "crever la peau des choses" en n'etant spectacle de Rien. 
"La création d'une oeuvre, c'est la creation d'un monde" (Kan. dinsky). Au meme moment Planck decrète que la vision est un réactif fortuit très limité et la physique nucléaire pose l'équation irreprésentable de la masse et de l'energie. Mais l'artiste procède à rebours du scientifique en restituant au connu sa part d'inconnu et à la veille de la seconde guerre mondiale, le Moi s'efface devant le tout du monde. Les galaxies de Newman, l'espace même qui naît de la pulvérisation de la representation, dans la folie dionysiaque du geste dansé. "Je suis la nature," dit Pollock. Question posée à l'origine et le mot "commencement" est alors susceptible d'une multiplicité de sens. Les peintres non figuratifs des années cinquante ont voulu "retourner le paysage comme un gant" (Messagier), révéler l'apparition brute du monde, à travers ses nodosités, ses pores, les fibres de la matière.

Ecouter le geste du peintre, dit Merleau-Ponty, c'est capter les paroles sourdes que l'etre murmure, ce logos silencieux que les "voies du silence" peuvent délivrer en réalisant ce que la parole ne peut faire: une ouverture au monde sans concept. Ce qui suppose que le geste du peintre entretient avec le monde un rapport d'harmonie pre-etabli mais aussi qu'une méditation intense doit précéder cette délivrance. "Rien ne suggère comme le silence le sentiment des espaces illimites" (Bachelard).

Comme Van Gogh qui réfléchissait jour et nuit avant de peindre furieusement, Masson précise qu'une méditation intense doit précéder la rapiditée fulgurante d'exécution, de même que ce n'est qu'après un long apprentissage que le disciple dans la pratique ZEN du tir à l'arc parvient au seuil de l'art sans art. Lapoujade prend même l'exemple très prosaique de la cafetièe. Le peintre "existe" la cafetière jusqu'à la devenir en la décantant de tous ses graphismes habituels.

Voyants, visionnaires, ces peintres ne sont pourtant pas mystiquement ivres de leur objet. Il faut revenir aux ecrits de Kandinsky, Malevitch, Mondrian ou meme ceux de Newman, Lapoujade. Dans son hommage à Sima, Roger Gilbert-Lecomte révèlait la nature de cette lente progression vers l'intuition de l'Unite:

Pour explorer ce lieu du monde qui tient dans l'espace sans dimensions, entre le point voyant de l'oeil et l'ecran de la paupière, il faut une "ascension entendue comme ascèse," une épuration des souvenirs des cadavres sensoriels. Jusqu'à ces paysages de l'ame ou arbres, pierres, cristaux, nous rendent enfin perceptibles le silence du monde, le cristal 
du temps."

Qu'on ne tente donc pas de s'y rendre d'un saut en supprimant les stades qui y conduisent. L'exotisme de l'universel ne vous prend pas a la gorge directement. Les visionnaires sont assez rares parmi les peintres (Ensor, Bosh, Brueghel), et les artistes rétiniens (Werner, Bonnard, Monet, Seurat. . .) plus nombreux. Et il est vrai que notre vision du visionnaire est essentiellement négative, associée à l'idée de l'hallucination comme une pathologie de l'esprit.

Les lumières de Aufklarungcontre les ténèbres de l'illumination. Il faudrait revenir sur le role d'intuition, de la vision dans la démarche scientifique afin de dépasser certaines contradictions contestables.

On mesure bien aussi, ce qu'il fallut d' "epoche" successives, de radicalisation, d'efforts dans le renouvellement du langage dans le domaine de la musique. Conscience tragique des langages traditionnels d'abord avec le dodécaphonisme qui procédait à une organisation intentionnelle des douze sons dans la gamme chromatique, contemporaine a la fois de la relativité et de la naissance des abstractions.

Ce fut ensuite le "Silence," la jouissance du son isolé avec J. Cage; le retour au prémusical, à travers l'appareillage complexe de la musique électronique, ultime étape de la désintégration de la tonalité.

Et puis ce sont les convergences étonnantes qui nous paraissent devoir être soulignées, nous dirons que ce mythe d'une musique absolue déhistorisée n'est pas neuf. Il révèle à quel point, la question du retour à l'origine en musique comme en peinture, ce désir de réintégrer le temps historique dans le temps cosmique n'est en rien un aveu d'impuissance devant les crises répétées de la science. Mais tout au contraire la révelation d'un point aveugle au coeur du disoours scientifique puisque "tout ce que je sais du monde même par la science je le sais à partir d'une vue mienne sans laquelle les symboles de la science ne voudraient rien dire..$^{3 n}$

On pourra toujours discuter de la probabilité d'une expérience antéprédicative, alors que le geste de l'artiste est toujours informe et

${ }^{3} \mathrm{M}$. Merleau-Ponty. Le visible et l'invisibie. 
jamais délivré de l'artifice. De ce fond d'experience muette et solitaire dont parle Merleau-Ponty a propos de Cézanne, de cette couleur de Rothko qui buvarde sur les bords et rend visible l'opacité du commencement du monde: Davantage que le retour à la conscience donatrice de sens originaire, c'est la question de l'élévation du regard, de la parole à un degré poètique qui nous semble intéressant.

Ou selon l'image de Pierre-Jean Jouve, le moment où l'homme mele au tronc d'arbre ou menace par les murs qui se rapprochent de lui, répond par le coup d'aile. Et cela, pour l'artiste dans le vif d'un destin personnelque seuls des termes métaphoriques permettraient d'exprimer: suicide, conversion, holocauste intérieur. La poésie contemporaine se reconnait d'ailleurs à ceci qu'elle cherche à se frayer un chemin où s'ecrit un impossible a écrire, à ceci qu'elle y fait constamment retour et ne consent jamais à le tenir pour rien.

On ne s'installe pas dans la sérénité d'une pensée extasée mais on y conjugue les contraires: le "Rien fecond" de Saint John Perse, "l'éclair qui dure" de R. Char, "La chaleur vacante" de Dubochet, la "limite illimitée" de Jacottet. Inutilite du commentaire explicatif, la ou il suffirait de montrer en écho, certaines toiles. Celles de Sima par exemple en résonnance avec l'oeuvre poetique de Gilben-Lecomte, Michaux ou Char, lorsqu'ils nous présentent un cosmos figure à l'etat naissant mais où donne déjà la pensée d'un dieu géomètre et où la couleur en paraît jamais captive sous la trame chiffree.

\section{Le deplacement du "regard"}

Exotisme de l'in-humain, de l'universel qui est aussi lié à une interrogation sur l'origine du geste et des cadres dans lesquels s'inscrit le geste.

Toute une reflexion sur la praxis des peintres, musiciens, poètes prend son départ. Dans la forme rectangulaire du tableau d'abord puisque celui-ci cesse d'être une règle du bon sens. Lorsque Viallat cherche l'envers, le refoule, le support de la peinture, la peinture n'est cette fois ni nature, ni religion, ni intériorité mais question posée à la peinture, a ses constituants, en ne négligeant aucun effet (platitude de la toile, plis du drap, transparence épaisseur, expériences de solarisation, détérioration, superposition) art d'entêtement où la répétition finit par produire de la difference. 
D'autres pousseront la convention jusqu'à l'absurde abusant du cadre, et feront surgir le scandale par un conformisme excessif, outre. Une toile vierge cernée avec insistance, cadres vides comme nos encadrements sociaux.

On reconnaît là cette autre forme d'exotisme inauguree par le Dadaisme et Marcel Duchamp. Exotisme du regard froid et non plus de la clairvoyance. Défi à la notion de valeur, ou exaltation d'un choix individuel et souverain qui n'a de comptes à rendre à personne, le readymade a fait de Duchamp l'artiste le plus populaire, même si, comme le lui reprochera Joseph Beuys il n'a peut-etre pas compris lui-même son propre apport ${ }^{4}$. Ce sont les "regardeurs" qui font désormais la peinture et nous sommes sans doute à nouveau ramenés à la conversion husserliennee du regard. Puisqu'il y est question du degré zéro de l'art et de cette experience nodale theatrale de la saisie de l'objet par le spectateur.

On pense à l'attitude de Tony Smith sur les autoroutes, ou à ces ready-made devenus paradoxalement de véritables fétiches voués à un culte attentif depuis leur consécration, conduisant à des invocations récurrentes chaque fois qu'il est question des frontieres.

Et quand J. Beuys prefere agir en sourcier pour retrouver l'energie des objets, il n'est pas si eloigné de ces peintres qui prétendent retourner le paysage comme un gant, à travers l'épiphanie du sensible.

Pourtant l'artiste, là, c'est l'anti-Cézanne si on ramène celui-ci à la formule "refaire cent fois le devant de la chemise." La secheresse de l'idée lancée une fois pour toutes, le domaine du coup, du jeu, de la "cosa mentale." L'artiste est celui qui refuse d'apparaître comme un créateur idéal, un demiurge consciencieux. En choisissant soit la réhabilitation de la matiere, la promotion de l'abject, soit au contraire la survalorisation du concept.

Dans un cas, désacraliser l'oeuvre d'art jusqu'à la promotion des gestes ratés, des spectacles honteux, c'est montrer à une culture qui ne veut pas la voir l'existence de la pourriture et de la mort. Ainsi Dubuffet conteste-t-il l'aristotélisme qui est en nous. "J'aime l'embryonnaire, le mal façonne, l'imparfait." Au moins, depuis les Merzbilder de Schwitters, l'art

${ }^{4} L e$ silence de Duchamp est surestime, piece intitulee ainsi par J. Beuys. 
n'a cessé de s'intéresser aux déchets, aux détritus à ce qui choit de notre sociéte. Et qui présente dans les oeuvres de Rauschenberg une certaine splendeur. Les artistes americains ont été d'ailleurs très loin dans cette réflexion, entrant dans les pieges de l'enfer social pour les désamorcer. Toute une generation d'artistes, comme Spoerri, fixe son époque à coup d'épluche-légumes; dadaïse en reprenant à son compte la phrase de Tzara (1919): "Nous sommes directeurs de cirque et nous soufflons dans les vents de foire."

Or, la contestation de l'ancienne conception du geste createur, l'affirmation de l'inutilite du coup de patte sont manifestes egalement chez les artistes conceptuels. Nous sommes la dans une situation ou la disparition de l'oeuvre et la survalorisation du concept augmentent la portée de la signature. Fait singulier, spécifique de la constitution d'une grande collection d'art conceptuel, le collectionneur --le vrai-- à ne pas confondre avec fétichiste qui réagit bêtement aux sensations plastiques, achete et possede les oeuvres de Flavin, Nauman, Irvin sur plans, sous forme d'un cahier des charges donnant un certain nombre de consignes en vue de la réalisation de l'oeuvre. Et comme le pouvoir de l'imagination du vrai collectionneur repose sur la connaissance préalable qu'il a de l'oeuvre de l'artiste, c'est bien l'invention d'un espace entre les actes, (acte d'ecriture et acte de transformation de la matiere) qui s'inscrit dans ce rétrécissement du champ visuel et cette progression de l'invisible.

L'appréciation esthétique est secondaire et le fossé s'accroit entre le public non averti et la communaute des artistes, collectionneurs, marchands et critiques d'art.

Distance philosophique aussi entre langage et matiere. Signer c'est bien ici faire un signe à la mort puisque la signature est un acte d'expression du sujet qui engage par dela la mort celui qui le commet.

On voit qu'il y a plusieurs voies possibles et plusieurs manières de rencontrer la mort, les morts et que si un jour, les artistes ont eu le sentiment de ne pas faire le meme métier que Giotto ou Piera della Francesca, c'est a partir de Duchamp et pour une part seulement d'entre eux; là où le questionnement l'emporte, là ou selon l'expression de Michel Fried, la théatralité domine car regardeurs (ou auditeurs placés au coeur de nombreuses sources sonores de la musique in situ) sont désormais ceux sans lesquels il ne pourrait y avoir d'oeuvre. Stockausen dans Momente integre même les applaudissements du public. 
Mais ce sens de la question de fond, question commune posée à l'origine du geste createur, ou geste tout court, comme dit, Artaud, aux sources plastiques, actives, incantatoires d'un langage producteur d'effets musicaux et de non sens, impose de toutes façons autre chose qu'une lecture lineaire qui s'inscrirait dans l'ordre symbolique d'une langue de communication ou de représentation.

Il y a là sans doute, comme une conquête douloureuse dont on peut suivre quelques étapes: famille, école, parricide. Puisqu'il ne va pas de soi, comme l'écrit Michel Serres, que nous renoncions à une parole qui s'échange entre nous comme etant illusoire à notre déséquilibre, orthopédie labile et flottante qui remplit les vides. Là où l'art aurait ce pouvoir de nommer ce vide, de lui assigner un lieu dans l'espace, il instaurera ou n'instaurera pas un vertige selon notre plus ou moins grande aptitude à accepter "la perception aiguê et immédiate d'une incomprehensibilité éternellens. Et ce coup d'aile jouvien peut-il être enseigné à l'école?

\section{"Culture artintique"}

Comprehension des oeuvres d'art, crise des institutions.

Si toute notre culture repose sur la certitude d'un univers plein et d'un homme cohérent au savoir infaillible, la culture artistique nous apparait d'abord comme un héritage, un bien de famille, disait Bourdieu, qui s'accompagne du sentiment d'appartenance à une classe qui trouve sa justification d'exister dans son harmonie, à un monde qui a produit des Beethoven et des Mozart et reproduit continuellement des gens capables de les jouer et de les gouter. La culture artistique s'acquiert là ou préexiste une image de soi, une aptitude a produire un monde contre nature, à transgresser les lois de la pesanteur en danse, à renoncer à la jouissance grossière des mélodies faciles, musique légère, exécutions futiles, racoleuses, tape-à-l'oeil, sirupeuses. Que le rapport bourgeois à la culture soit lointain ou dissertatif ou plus technique, la sublimation artistique "enferme nécessairement l'affirmation de la sublimite" de ceux qui savent rechercher les plaisirs gratuits libres ${ }^{6}$.

\footnotetext{
${ }^{5}$ V. Segalen. Essais sur l'exotisme.

- P. Bourdieu. La distinction.
} 
Au-delà de la famille, commence l'école. On connait l'importance de la corrélation entre le capital scolaire et le capital culturel toujours un peu paradoxale quand il s'agit de domaines que l'ecole enseigne peu. Le gonflement des effectifs dans le lycke est accompagné d'une modification du comportement culturel: le nombre de visiteurs dans les musées est passé au cours des sept dernières annees de neuf à douze millions. Même constat pour les festivals dont la fréquentation est aussi fortement correlée à la détention d'un capital scolaire. On peut aussi saisir la relation entre l'enseignement secondaire et la lecture en étudiant la diversite des titres des livres choisis par les lycéens. Reste que les jeunes des LP manifestent en valorisant fortement les traditions de leur sexe (lecture de publications sportives et de magazines feminins) alors que les lyceens disent appartenir a "la culture" et que la culture leur appartient. Il y a bien de la différence entre la musique écoutée et la musique dansée, la mode ou la distance qu'on prend par rapport à la mode.

Pourtant, même l'augmentation du public dans les musées ou les salles de concert ne permet pas de dire que la culture artistique concerne plus d'individus si par ailleurs on ne se donne pas les moyens d'une réflexion sur l'impact provoqué par les oeuvres sur le public. "Sémantiquement autonome" et "contenu sédimenté l'oeuvre d'art pourrait être considéree comme ce qui joue ses themes multiples sur les instruments de l'ame. L'école nous apprendrait d'abord à entendre les propos sensés de Porbus dans Le chef d'oeuvre inconnu de Balzac. Au discours exalté sur l'esthétique de Frenhofer, il repond: "Travaillez; les peintres ne doivent travailler que les brosses a la main!" Et même si lancienne conception du geste créateur est aujourd'huicontestée, la compréhension d'une oeuvre commence par son approche technique.

Mais si chanter, déchiffrer une partition est en partie un décodage et si la compréhension esthétique relève d'une compétence de lecteur, il est aise de montrer qu'elle ne réside pas seulement dans ce décodage, ni davantage dans un vécu émotif bien qu'elle s'accompagne de ce vécu jusque dans le détoumement de l'émotion. Pas plus qu'elle ne réside dans les gestes ou les discours qui en sont le contrepoint parlé et qui tissent autour de l'oeuvre un jeu d'allusions lettrées habiles a masquer certaines ignorances.

Si compréhension de l'oeuvre il y a, il s'agirait plutôt de "cette accession immédiate à une certaine intelligibilité mais non par les voies 
compliquees du raisonnement, par cette espace de heurt en pleine figure que nous inflige la prehension d'une configuration sensible", (LeviStrauss). Ce que Kandinsky appelait la saisie de la nécessité intérieure d'une oeuvre, concept dont on pourrait trouver les origines chez Goethe, Schiller, ou Wagner.

La perversité méme de l'art, consiste à créer des tâches impossibles à maîtriser par les voies du raisonnement ou par nos fonctions normales. Notamment l'essence de la rigueur artistique refuse la moindre distinction entre la signification d'eléments constitutifs et impose la saisie de la structure globale d'une oeuvre en dépassant le contexte des phrases musicales, en transcendant les fragmentations pour saisir l'oeuvre comme un tout indivisible. Cette experience intime d'un sens, c'est un peu comme si, musiciens, nous trouvions enfin l'expression juste pour un morceau qu'on répétait sans que pour autant la recherche s'arrête là.

Il ne s'agit pas de la conformité à un modèle préexistant puisque l'oeuvre garde sa liberté d'echapper a toutes les interprétations mais l'evaluation de la justesse impérieuse d'une performance. Le "Cela devait être ainsi" de Wittgenstein.

Il y a un ordre éminemment constructeur des processus primaires mais il y a aussi et surtout la mobilisation instantanée de toute ma culture --car pour comprendre une phrase musicale c'est toute la culture que nous devons mobiliser dans l'instant. Il ne s'agit pas de démontrer l'inutilite de l'enseignement de l'art en evoquant comme Lapoujade "les mécanismes de la fascination", et la sociologie nous a mis en garde contre cette tentation de l'esthète à reconnaître comme seul légitime le rapport a la culture qui porte le moins de traces visibles de sa genèse, en n'ayant rien d'appris, ni d'apprêté, ni de scolaire ou de livresque et qui manifeste par l'aisance et le naturel que la vraie culture est nature, nouveau mystère de l'immaculée conception (cf. Bourdieu).

Si la compréhension esthétique est un certain plaisir à mesurer une distance, une altérité, une incomprehensibilité et à les déguster, plaisir qui nait sur le fond d'une mobilisation de la culture toute entière, musique, peinture, poésie sont des formes artistiques interactives, et c'est sans doute parce que les institutions chargées de transmettre l'héritage artistique ont mal compris cette interactivite qu'elles connaissent une crise profonde. 
Crise de l'IRCAM, des écoles d'art, des conservatoires qui prennent conscience depuis l'enquete publique de 1983 d'un fosse infranchissable par rapport à la réalité de départ de ceux qu'ils forment entre les instruments "ideaux" et ceux que choisissent plus tard les adolescents. L'envers d'une politique sélective, copiant le modèle scolaire, c'est l'importance du taux d'échec: $38 \%$ des élèves de onze ans se sont arrêtés ou repliés vers d'autres lieux. C'est aussi le handicap relatif de la France, ou son enseignement trop technique et une méconnaissance du repertoire contemporain ou extra-européen relevent une absence totale d'ouverture par contraste avec d'autres pays europeens; moins d'artistes, moins d'enseignants qualifies, un public socialement peu diversifie.

Devant la montee de la contestation et des discours qui ressassent la question de la crise institutionnelle, la tentation est finalement grande d'intégrer dans les contenus d'enseignement seuls incriminés tout et n'importe quoi, au nom d'un principe de representativité qui ferait de ces institutions enfin le reflet de pratiques moyennes. De faire du thème nihiliste de Duchamp qui radicalise à l'extrême la question de la précarité des valeurs esthétiques non pas une exigence du regard ou de l'oreille mais une véritable idéologie du mineur qui se caracteéise en gros par trois postulats: la revendication d'une totale liberté dans le choix des styles et des matériaux, le constat de la fin des avant-gardes et soupfon porté sur l'ensemble de l'art, enfin le populisme, ou l'idée d'un recours aux éléments culturels populaires comme force de subversion (il ne serait pac inutile de rappeler la généalogie de la mythologie du populaire dans les pays germaniques).

Cette idéologie, on ne peut la repérer chez ces musiciens qui s'appuient sur les traditions du music-hall pour liquider l'ecole de Vienne ou chez les peintres de la figuration libre qui soumettent la peinture à l'esthétique de la bande dessinée.

La musique de Mahler compte sur l'inférieur mais ce recours aux ritournelles, aux fanfares militaires ne renvoie pas à quelque nostalgie de l'authenticité populaire. Il s'agit plutot, comme le suggerait Kandinsky, de jouer cette contamination du mineur par le majeur, de relever la fin de l'innocence.

\section{Quelle pedagogie?}

Sans doute est-ce la un probleme pedagogique essentiel. 
"Tiedeur, ${ }^{\text {n }}$ "Bouillon de culture" ${ }^{n 8}$, acceptation pure et simple "d'une sous culture de masse, ${ }^{\text {9" }}$ "indigestion, ${ }^{10 \text { " }}$ "imagologie, ${ }^{11 \text { " }}$ goat du "sensationnel et de l'exotisme touristique, ${ }^{12 n}$ les qualificatifs critiques ne manquent pas.

S'il y a des oeuvres qui, quelle que soit l'epoque, quelle que soit la culture--posent de ces questions essentielles et incitent à des rêveries essentielles, l'un des rôles de la pédagogie ne doit-il pas être d'explorer l'art ancien et moderne en sourcier et de réhabiliter une vigilance critique à l'égard des lignes de démarcation? quitte à enseigner ensuite à les perturber.

L'idée du Blaue Reiter ou du Bauhaus de mettre en résonance des oeuvres, proposant à une sonoritee une contre sonorite, l'idée aussi d'une approche synthétique de l'art, quatre vingts ans après Kandinsky et Marc, paraissent encore très revolutionnaires. Segalen disait qu'on formait le jugement artistique en confrontant des objets et non en leur accolant des commentaires explicatifs. On pourrait aussi imaginer un nouveau modeèe de musée qui renouerait avec les anciens cabinets de curiosité antérieurs au cartésianisme. Mais comment réveiller le désir créateur?

Par une pédagogie de la découverte d'abord et la suspension de la parole: Il est étonnant de voir à quel point l'éducation scolaire est encore sous la dépendance du langage, du commentaire des archives, de ces livres d'histoire et de géographie dont Segalen disait qu'ils tuaient l'exotisme spontane de l'enfant. "Le triomphe du Verbe fut une catastrophe perceptive" écrivait $M$. Serres. Le theme n'est pas neuf et on a souvent opposé en alternative au dictionnaire encyclopédique des choses une contre-pédagogie du Voir et de l'Entendre.

`V. Segalen. Essais sur l'exotisme.

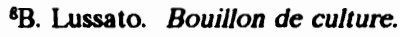

'Scarpetta.

10V. Janek.

"M. Kundera.

12 V. Segalen. 
Mais tout le problème est là: comment les adultes montreraientils aux enfants un monde qu'ils ont perdu? Pour percevoir les mille nuances de gris entre le noir et le blanc que nous ne savons plus enregistrer, pour former une oreille explorative imaginaire, pour retrouver la douceur, la rugosité, la lenteur, la vitesse des mots, ce procès semiotique sous le symbolique qui donnerait aux enfants le goat du palpiter, du renaître, du vaciller avec ce qu'ils voient, entendent, touchent, pour sauver l'alchimiste sous l'ingenieur il ne faut pas être toujours pret à repondre. La parole tue le désir avant qu'il ne puisse éclore. Comment redonner le goat du vertige devant ce qui glisse sous nos pas?

Les vraies sensations d'exotisme commencent quand on renonce au savoir infaillible, lorsqu'on accepte l'altérité comme une objectivité éternellement incompréhensible et ce n'est sans doute pas en décrétant péremptoirement devant les enfants, "ceci est beau ou majeur," "ceci est laid ou mineur!" que nous contribueronsà former le jugement esthétique. Tout au contraire.

A l'inverse des arrêts de la logique, (et c'est ce que nous avons appris du dadaïsme ou du surrealisme), le gout de la subversion, du deplacement du regard aussi bien sur le lointain que sur le quotidien, que Michaux appelait le merveilleux normal, doit entrer à l'école.

C'est toute l'efficacité d'une pensée latérale en pédagogie dont la fecondité pourrait aussi être soulignée en sciences dans l'émergence des idées neuves.

Sans proposer comme Dubuffet des instituts de déculturation où des gymnases nihilistes ou l'on apprendrait que le savoir n'est pas infaillible, nous pouvons imaginer dans le contexte de nos grandes societés industrielles atomisees des espaces et des moments de Silence pour voir, entendre, rever, explorer, deplacer, subvertir. On ne travaillerait pas à former seulement des artistes mais aussi des philosophes et des savants.

A l'inverse de cette longue péeriode de terreur intellectuelle ou les hommes capables de vraies questions ou de jeux d'esprit, les rares génies capables de grande profondeur ou de grande légèreté font figure de miraculés.

A ceux qui nous objecteraient que ce n'est pas au moment ou se construit le savoir qu'il faut montrer sa fragilité, qu'on ne donne pas à des 
enfants le gout du vertige et des grandes questions sans risque, nous dirons que l'artiste tire son oeuvre des profondeurs les plus intimes de la vie. Si le monde moderne accule l'art à l'isolement, l'artiste sent qu'il devrait au contraire trouver sa place au coeur de l'etre commun, et qu'il faut apprendre aux enfants à fréquenter les oeuvres de manière ordinaire.

"L'enjeu véritable n'est alors pas l'aspect extérieur de l'oeuvre mais la fonction de l'art. Et pour que l'art fonctionne", il faut précisément qu'il se distende jusqu'à atteindre cette frontière au delà de laquelle il cesse d'être de l'art (....). L'art moderne naît d'une grande simplicité humaine (. . .). S'il doit continuer, il devra inventer des manières toujours nouvelles d'echapper à ces limites qui lui sont sans cesse opposées. Et cela se fera sans beaucoup de bruit. Ce ne sera pas une tempete qui emporterait les digues mais plutôt une infiltration. L'art s'immiscera dans le monde moderne par des fissures. C'est par sa simplicité qu'il touchera le coeur des hommes. ${ }^{13 n}$

\section{MARIE-HEILENE POPEILARD}

L'expose ci-dessus a été présenté au Colloque de La Rochelle, "Le métier d'instruire," organise sous la direction de Laurence Cornu avec le concours du College international de philosophie, du 15 au 16 mai 1990.

"J. Chalupecky. Postface alox histoires, 1984. 